\title{
OFF-SHELL NN POTENTIAL AND TRITON BINDING ENERGY"
}

\author{
Y. Song and R. Machleidt \\ Department of Physics, University of Idaho, Moscow, ID 83843, U.S.A. \\ SUMMARY AND CONCLUSIONS
}

The (nonlocal) Bonn-B potential predicts $8.0 \mathrm{MeV}$ binding energy for the triton (in a charge-dependent 34-channel Faddeev calculation) which is about $0.4 \mathrm{MeV}$ more than the predictions by local NN potentials. We pin down origin and size of the nonlocality in the Bonn potential, in analytic and numeric form. The nonlocality is due to the use of the correct off-shell Feynman amplitude of one-boson-exchange avoiding the commonly used onshell approximations which yield the local potentials. We also illustrate how this off-shell behavior leads to more binding energy. We emphasize that the increased binding energy is not due to on-shell differences (differences in the fit of the NN data or phase shifts). In particular, the Bonn-B potential reproduces accurately the $\epsilon_{1}$ mixing parameter up to $350 \mathrm{MeV}$ as determined in the recent Nijmegen multi-energy NN phase-shift analysis. Adding the relativistic effect from the relativistic nucleon propagators in the Faddeev equations, brings the Bonn-B result up to $8.2 \mathrm{MeV}$ triton binding [1]. This leaves a difference of only $0.3 \mathrm{MeV}$ to experiment, which may possibly be explained by refinements in the treatment of relativity and the inclusion of other nonlocalities (e. g., quark-gluon exchange at short range). Thus, it is conceivable that a realistic NN potential which describes the NN data up to $300 \mathrm{MeV}$ correctly may explain the triton binding energy without recourse to 3-N forces; relativity would play a major role for this result.

\section{INTRODUCTION}

Recently it has been shown that local $N N$ potentials lead to a triton binding energy of $7.62 \pm 0.01 \mathrm{MeV}[2]$, i. e., they underbind the triton by 0.86 $M e V$. On the other hand, it is well known that the nuclear force must contain nonlocalities, since any more fundamental mechanism for creating the

\footnotetext{
${ }^{1}$ Contributed Paper submitted to the 14th International Conference on Few Body Problems in Physics, Williamsburg, Virginia, USA, May 26-31, 1994.
} 
nuclear force generates a nonlocal interaction. Such nonlocalities may increase binding energy predictions for systems of three or more nucleons. An example is the Bonn-B potential [3] which predicts $7.97 \mathrm{MeV}$ binding energy for the triton in a charge-dependent 34-channel Faddeev calculation [4].

Before one can pin down off-shell effects, it is absolutely crucial to make sure that the potential under consideration reproduces accurately the onshell NN data. Of particular importance is here the $\epsilon_{1}$ mixing parameter which is a measure for the on-shell tensor force strength. Figure 1 shows that the the Bonn-B potentials predicts $\epsilon_{1}$ in accurate agreement with the most recent Nijmegen multi-energy NN phase-shift analysis [6].

\section{OFF-SHELL POTENTIAL AND NN $t$-MATRIX}

Predictions for NN observables may be based upon the on-shell twonucleon $t$-matrix derived from a given NN potential $V$. The off-shell NN $t$-matrix is input for momentum-space Faddeev calculations of the threenucleon system. The calculation of the $t$-matrix always involves the NN potential on- and off-(the-energy-)shell. We illustrate this for the example of the partial-wave $t$-matrix, $t_{L^{\prime} L}^{J S T}$, in the ${ }^{3} S_{1}$ two-nucleon state, which is given by

$$
\begin{aligned}
t_{00}^{110}\left(q^{\prime}, q ; E\right)= & V_{00}^{110}\left(q^{\prime}, q\right)-\int_{0}^{\infty} k^{2} d k V_{00}^{110}\left(q^{\prime}, k\right) \frac{M}{k^{2}-M E-i \epsilon} t_{00}^{110}(k, q ; E) \\
& -\int_{0}^{\infty} k^{2} d k V_{02}^{110}\left(q^{\prime}, k\right) \frac{M}{k^{2}-M E-i \epsilon} t_{20}^{110}(k, q ; E)
\end{aligned}
$$

For free-space NN scattering, $E=q_{0}^{2} / M$ with $M$ the nucleon mass and $q_{0}$ the c.m. on-shell momentum which is related to the lab. energy by $E_{l a b}=2 q_{0}^{2} / M$. Notice that in the integral terms the potential contributes essentially off-shell. In general, the second integral which involves $V_{02}^{110}$ (tensor force) is much larger than the first integral that involves $V_{00}^{110}$ (central force). Therefore, we will focus here on the second integral term and the tensor force.

In Fig. 2, we show the ${ }^{3} S_{1^{-}}{ }^{3} D_{1}$ potential matrix element, $-V_{02}^{110}\left(q_{0}, k\right)$, for Paris [5] and Bonn [3]. The momentum $q_{0}$ is held fixed at $153 \mathrm{MeV}$ which corresponds to a lab. energy of $50 \mathrm{MeV}$. The abscissa, $k$, is the variable over which the integrations are performed in the above equation. It is seen that, particularly for large off-shell momenta, the Bonn-B potential is substantially smaller than the Paris potential: the Bonn-B potential has clearly a weaker 
off-shell tensor force than the Paris potential. As a consequence of this, the Paris potential produces a much larger integral term in Eq. (1) than the Bonn potential; and it is this integral term that is subject to quenching in few- and many-body calculations. In the three-body Faddeev equations, the $t$-matrix is fully off-shell and $E$ is negative; the negative $E$ reduces the magnitude of the integral term. The larger the term, the larger the quenching. Since this integral term is attractive, the quenching is a repulsive effect. Thus, large off-shell potentials, implying a large integral term, yield less attraction in three- and many-body problems. This explains why the Paris potential predicts less triton binding energy than the Bonn potential.

\section{THE ORIGIN OF OFF-SHELL DIFFERENCES}

The Bonn potential is defined in terms of the full, relativistic Feynman amplitudes of one-boson-exchange, which are non-local expressions. On the other hand, the Paris potential is (apart from a $\mathbf{p}^{2}$-term in the central force) a local potential. The local approximation of the pion tensor potential, which

is used in the Paris potential as well as in any other local $r$-space potential, has the familiar form,

$$
{ }^{\pi} \tilde{V}_{T}(r)=-\frac{g_{\pi}^{2}}{4 \pi}\left(\frac{m_{\pi}}{2 M}\right)^{2}\left(1+\frac{3}{m_{\pi} r}+\frac{3}{\left(m_{\pi} r\right)^{2}}\right) \frac{e^{-m_{\pi} r}}{r} S_{12} .
$$

The transformation of this local potential into momentum space yields for the ${ }^{3} S_{1^{-}}{ }^{3} D_{1}$ amplitude

$$
{ }^{\pi} \tilde{V}_{02}^{110}\left(q_{0}, k\right)=-\frac{g_{\pi}^{2}}{4 \pi} \frac{\sqrt{8}}{4 \pi M^{2} q_{0} k}\left[q_{0}^{2} Q_{2}(z)-2 q_{0} k Q_{1}(z)+k^{2} Q_{0}(z)\right]
$$

with $Q_{L}$ Legendre funct. of the 2 . kind and $z \equiv\left(q_{0}^{2}+k^{2}+m_{\pi}^{2}\right) /\left(2 q_{0} k\right)$. The original ${ }^{3} S_{1^{-}}{ }^{3} D_{1}$ transition potential as it results from the relativistic one-pion-exchange Feynman amplitude is

$$
\begin{aligned}
{ }^{\pi} V_{02}^{110}\left(q_{0}, k\right)= & -\frac{g_{\pi}^{2}}{4 \pi} \frac{\sqrt{8}}{4 \pi M^{2} q_{0} k}\left[\left(E_{q_{0}}-M\right)\left(E_{k}+M\right) Q_{2}(z)-2 q_{0} k Q_{1}(z)\right. \\
& \left.+\left(E_{q_{0}}+M\right)\left(E_{k}-M\right) Q_{0}(z)\right]
\end{aligned}
$$


with $E_{q_{0}} \equiv \sqrt{M^{2}+q_{0}^{2}}$ and $E_{k} \equiv \sqrt{M^{2}+k^{2}}$. Expanding these roots in terms of $q_{0}^{2} / M$ and $k^{2} / M$ yields

$$
\begin{aligned}
{ }^{\pi} V_{02}^{110}\left(q_{0}, k\right) \approx & -\frac{g_{\pi}^{2}}{4 \pi} \frac{\sqrt{8}}{4 \pi M^{2} q_{0} k}\left[\left(q_{0}^{2}+\frac{q_{0}^{2} k^{2}}{4 M^{2}}-\frac{q_{0}^{4}}{4 M^{2}} \cdots\right) Q_{2}(z)-2 q_{0} k Q_{1}(z)\right. \\
& \left.+\left(k^{2}+\frac{q_{0}^{2} k^{2}}{4 M^{2}}-\frac{k^{4}}{4 M^{2}} \cdots\right) Q_{0}(z)\right]
\end{aligned}
$$

Keeping terms up to momentum squared, leads to the local approximation Eq. (3). The largest term in the next order is $-k^{2} /\left(4 M^{2}\right) Q_{0}(z)$ which damps the tensor potential off-shell.

The thin short-dashed curve in Fig. 2 shows what is obtained when in the Bonn-B potential the pion tensor force is made local and the dotted curve results when both $\pi$ and $\rho$ are local. The latter curve is very close the Paris curve (thick long-dashed line), which explains the origin for the differences between the Paris and Bonn potentials. Another source for nonlocality in the Bonn potential is the factor $M / \sqrt{E_{q_{0}} E_{k}}$ which is applied to the Feynman amplitude to define the potential. The nonlocality created by this factor has been investigated in great detail by Glöckle and Witala [7]. This factor is included in the solid, short-dashed, and dotted curves in Fig. 2. When this factor is omitted in the latter case, the widely-spaced dots are obtained, which demonstrates an additional source of nonlocality in the Bonn potential.

This work was supported in part by NSF-Grant PHY-9211607.

\section{REFERENCES}

1. F. Sammarruca, D. P. Xu, and R. Machleidt, Phys. Rev. C 46, 1636 (1992).

2. J. L. Friar et al., Phys. Lett. B311, 4 (1993).

3. R. Machleidt, Adv. Nucl. Phys. 19, 189 (1989).

4. D. Y. Song and R. Machleidt, unpublished.

5. M. Lacombe et al., Phys. Rev. C 21, 861 (1980).

6. V. G. J. Stoks et al., Phys. Rev. C 48, 792 (1993).

7. W. Glöckle and H. Kamada, Nonlocalities in NN forces defined through the Blankenbecler-Sugar equation, preprint, University of Bochum (1994). 


\section{Figure Captions}

Figure 1. The $\epsilon_{1}$ mixing parameter below $350 \mathrm{MeV}$ as predicted by the Bonn-B [3] (solid line) and the Paris [5] (dashed) potentials. The solid dots represent the Nijmegen multi-energy NN phase shift analysis [6].

Figure 2. Half off-shell ${ }^{3} S_{1^{-}}{ }^{3} D_{1}$ transition potential of the Bonn-B [3] (solid line) and the Paris [5] (thick dashed line) potentials. The thin shortdashed and dotted lines are obtained when in the Bonn-B potential the local approximation is used for the $\pi$ and for both $\pi$ and $\rho$, respectively. When in the latter case also the $M / \sqrt{E_{q_{0}} E_{k}}$ factor is left out, the widely-spaced dots are obtained. The solid dot is the on-shell point $\left(k=q_{0}\right)$ at which all curves are the same.

The figures are available upon request from MACHLEID@TAMALUIT.PHYS.UIDAHO.EDU

Please include your FAX number or mailing address with your request. 
This figure "fig1-1.png" is available in "png" format from: http://arxiv.org/ps/nucl-th/9403019v1 
This figure "fig1-2.png" is available in "png" format from: http://arxiv.org/ps/nucl-th/9403019v1 